\title{
Editorial
}

\section{Application of Cardiovascular Mechanics to Risk Stratification in End Stage Renal Disease}

\author{
Simon Ray \\ Department of Cardiology, University Hospitals of South Manchester, Manchester, UK
}

The study by Chiu et al. [1] examines 2 established non-invasive measures of cardiovascular mechanics in a population of patients undergoing maintenance haemodialysis. Global longitudinal strain (GLS) is a measure of myocardial deformation and pulse wave velocity (PWV) is a measure of aortic stiffness. Both GLS and PWV are recognised as predictors of mortality in end-stage kidney disease. Although not large (198 evaluable subjects) and restricted to a single centre, this is the largest series to combine both measures and it is worth emphasising that this was a high-risk population. Almost $40 \%$ were diabetic and more than a quarter had an existing diagnosis of coronary artery disease.

GLS using speckle tracking echocardiography has an expanding role in cardiology practice and has proved to be superior to left ventricular ejection fraction (LVEF) in predicting outcomes in situations as diverse as sepsis [2], heart failure with preserved ejection fraction [3] and late after cardiotoxic chemotherapy [4]. As such, it is part of the repertoire of many cardiology departments. Speckle tracking relies on reasonable greyscale echocardiographic image quality but has the attraction that analysis is semi-automated. The key to its superiority over LVEF is that it may be abnormal when LVEF is within range, especially in conditions associated with increased afterload and left ventricular hypertrophy where a normal ejection fraction does not necessarily equate to normal contractile function. It is also more reproducible. As a measure of vascular stiffness, PWV is often regarded as a functional assessment of vascular aging, and has a longer track record than GLS. It is proven to be associated with cardiovascular outcomes across a range of conditions, but although it is included in the current European guidelines for the management of hypertension [5], it is less widely used in routine cardiology practice.

Chui et al. [1] found that a more positive (more abnormal) GLS predicted cardiac death and major cardiac events but not all-cause mortality. However, the associations were not particularly strong and diabetes was a much stronger predictor of cardiac death than GLS. Age, increased PWV and indexed LV mass were the only predictors of all-cause mortality. At first sight, it is surprising that PWV predicted all-cause mortality but not cardiac mortality or major cardiac events, given its established predictive role in cardiac outcomes in end-stage kidney disease [6]. It is also perhaps surprising that the 2 parameters themselves demonstrated no association given the impact of aortic stiffness on ventricular loading and hence ventricular mechanics. Reduced (i.e. more positive) GLS reflects impairment of contraction of the subendocardial

\section{KARGER}

E-Mail karger@karger.com

www.karger.com/ajn
(C) 2016 S. Karger AG, Basel

0250-8095/16/0433-0141\$39.50/0
Simon Ray, MD, FRCP

Department of Cardiology

University Hospitals of South Manchester

Manchester M23 9LT (UK)

E-Mail Simon.ray@uhsm.nhs.uk 
muscle fibres in the left ventricle. These fibres are more susceptible to injury than the mid-wall or subepicardial fibres as a result of their more precarious blood supply, particularly in a hypertrophied or pressure overloaded ventricle. In this study, this is entirely consistent with the relationships of GLS with the left ventricular mass indexed for height and with systolic blood pressure.

In contrast Krishnasamy et al. [7] in a meticulous study of a population of 136 patients with CKD stages 3-4 found that GLS was related to LV mass but not systolic blood pressure, possibly as a result of a high prevalence of treated hypertension. GLS was, however, related to indices of uraemic toxicity and body mass index. More importantly, in this series, there was a relationship between PWV and GLS that remained significant despite adjustment for traditional risk factors and echocardiographic variables.

Chiu et al. [1] speculate that this discrepancy between the 2 populations might be related to the specifics of the metabolic milieu of the dialysis patient. They also suggest that GLS and PWV might provide complimentary prognostic information and could therefore be useful clinically, although this proposal has to be tempered with some important caveats. The first and most important of these is that both their study and that of Krishnasamy et al. [7] are relatively small samples of patients recruited from a single centre. If GLS is to be of practical use in the management of dialysis patients, then the results obtained by Chiu need to be broadly generalisable. As the authors acknowledge, the measurement of GLS is not always straightforward and it may not be as reproducible when performed outside the context of a single-centre research effort. There are also differences in the algorithms used by different manufacturers to track specular echoes within the myocardium, so that the results obtained on one system are not necessarily consistent with those of another. Nonetheless, it is important to acknowledge that LVEF has remained the common currency for the assessment of LV function for many years despite well-recognised concerns about reproducibility and load dependence.

The second key issue is that both studies are crosssectional. If GLS is to be clinically useful in renal patients, then it will be important to understand its evolution with the progression of the underlying disease and particularly with the establishment of dialysis. The metabolic milieu of the dialysis patient might alter GLS in the light of its association with uraemic toxins [7], as it could possibly modify the relationship between GLS and PWV [1].

A closely related question is the impact of medical treatment on GLS. If a subnormal GLS is used as a marker of increased cardiovascular risk, then what steps can be taken to reduce risk in a population where all risk factors should already be aggressively targeted and can a subsequent improvement in GLS be taken as an indication of risk reduction? Given the inherent variability in the technique, it seems unlikely that this will be feasible.

In summary, Chiu et al. [1] are to be congratulated for completing a meticulous clinical study in a high-risk patient group. Their study provides further evidence that GLS might be a useful technique in the risk stratification of patients with end-stage kidney disease, possibly in combination with PWV, but also emphasises the need for larger, ideally multicentre, studies to confirm their findings. Finally, both the current paper and that of Krishnasamy et al. [7] give emphasis to the complex interplay of metabolic and haemodynamic factors in determining cardiac risk in patients with advanced renal disease and the consequent complexity inherent in predicting outcomes.

\section{References}

1 Chiu D, Abidin N, Johnstone L, Chong M, Kataria V, Sewell J, et al: Novel approach to cardiovascular outcome prediction in haemodialysis patients. Am J Nephrol 2016;43:143152.

2 Palmieri V, Innocenti F, Guzzo A, Guerrini E, Vignaroli D, Pini R: Left ventricular systolic longitudinal function as predictor of outcome in patients with sepsis. Circ Cardiovasc Imaging 2015;8:e003865.

3 Sengeløv M, Jørgensen PG, Jensen JS, Bruun NE, Olsen FJ, Fritz-Hansen T, et al: Global longitudinal strain is a superior predictor of all-cause mortality in heart failure with re- duced ejection fraction. JACC Cardiovasc Imaging 2015;8:1351-1359.

4 Armstrong GT, Joshi VM, Ness KK, Marwick TH, Zhang N, Srivastava D, et al: Comprehensive echocardiographic detection of treatment-related cardiac dysfunction in adult survivors of childhood cancer: results from the St. Jude lifetime cohort study. J Am Coll Cardiol 2015;65:2511-2522.

5 Mancia G, Fagard R, Narkiewicz K, Redon J, Zanchetti A, Böhm M, et al: 2013 ESH/ESC guidelines for the management of arterial hypertension: the task force for the management of arterial hypertension of the European society of hypertension (ESH) and of the European society of cardiology (ESC). Eur Heart J 2013;34:2159-2219.

6 Blacher J, Safar ME, Guerin AP, Pannier B, Marchais SJ, London GM: Aortic pulse wave velocity index and mortality in endstage renal disease. Kidney Int 2003;63: 1852-1860.

7 Krishnasamy R, Hawley CM, Stanton T, Pascoe EM, Campbell KL, Rossi M, et al: Left ventricular global longitudinal strain is associated with cardiovascular risk factors and arterial stiffness in chronic kidney disease. BMC Nephrol 2015;16:106. 